This item was submitted to Loughborough's Research Repository by the author.

Items in Figshare are protected by copyright, with all rights reserved, unless otherwise indicated.

\title{
Direct detection of small molecules using a nano-molecular imprinted polymer receptor and a quartz crystal resonator driven at a fixed frequency and amplitude
}

\section{PLEASE CITE THE PUBLISHED VERSION}

https://doi.org/10.1016/j.bios.2020.112176

\section{PUBLISHER}

Elsevier BV

\section{VERSION}

AM (Accepted Manuscript)

\section{PUBLISHER STATEMENT}

This paper was accepted for publication in the journal Biosensors and Bioelectronics and the definitive published version is available at https://doi.org/10.1016/j.bios.2020.112176

\section{LICENCE}

CC BY-NC-ND 4.0

\section{REPOSITORY RECORD}

Guha, Arnab, Omar Sheej Ahmad, Antonio Guerreiro, Kal Karim, Niklas Sandström, Victor P Ostanin, Wouter van der Wijngaart, Sergey A Piletsky, and Sourav Ghosh. 2020. "Direct Detection of Small Molecules Using a Nano-molecular Imprinted Polymer Receptor and a Quartz Crystal Resonator Driven at a Fixed Frequency and Amplitude". figshare. https://hdl.handle.net/2134/12179283.v1. 


\section{Direct detection of small molecules using a nano-molecular imprinted polymer receptor and a quartz crystal resonator driven at a fixed frequency and amplitude}

Arnab Guha1, Omar Sheej Ahmad ${ }^{2,3}$, Antonio Guerreiro ${ }^{4}$, Kal Karim², Niklas Sandström Victor P Ostanin ${ }^{6}$, Wouter van der Wijngaart ${ }^{5}$, Sergey A Piletsky ${ }^{2}$, Sourav K Ghosh ${ }^{1 *}$

1 Centre for Biological Engineering, Loughborough University, Loughborough, UK

2 Department of Chemistry, University of Leicester, Leicester, UK

3 Department of Chemistry, College of Education for Pure Science, University of Mosul, Iraq 4 MIP Diagnostics, Leicester, UK

5 KTH Royal Institute of Technology, Stockholm, Sweden

6 Department of Chemistry, University of Cambridge, Cambridge, UK

${ }^{*}$ Corresponding author

\section{Abstract}

Small molecule detection is of wide interest in clinical and industrial applications. However, its accessibility is still limited as miniaturisation and system integration is challenged in reliability, costs and complexity. Here we combined a 14.3 MHz quartz crystal resonator (QCR), actuated and analysed using a fixed frequency drive (FFD) method, with a nanomolecular imprinted polymer for label-free, realtime detection of N-hexanoyl-L-homoserine lactone (199 Da), a gram-negative bacterial infection biomarker. The lowest concentration detected $(1 \mu \mathrm{M})$ without any optimisation was comparable with that of a BIAcore SPR system, an expensive laboratory gold standard, with significant enhancement in sensitivity and specificity beyond the state-ofthe-art QCR. The analytical formula-based FFD method can potentially allow a multiplexed "QCR-on-chip" technology, bringing a paradigm shift in speed, accessibility and affordability of small molecule detection.

Keywords: quartz crystal resonator (QCR); quartz crystal microbalance (QCM); fixed frequency drive; nanomolecular imprinted polymer (nano-MIP); acyl-homoserine lactone (AHL)

\section{Introduction: Small Molecular Detection}

With advancements in analytical technologies in the last decades, small molecule analytes, mostly with molecular weight less than $1000 \mathrm{Da}$, have gained increased interest in the fields of healthcare diagnosis and treatment, biosecurity, and food and environmental monitoring (Gooding, 2006; Powers and Riekeberg, 2017; Shankaran et al., 2007). In healthcare, small molecular markers or biomarkers, such as metabolites, enzymes, and peptides derived from disease-specific proteins, have been explored widely in diagnosis and assessment of therapeutic intervention for chronic diseases like cancer, Alzheimer's disease and chronic kidney disease (Grams et al., 2018; Holliger and Hudson, 2005; Kim and Legido-Quigley, 2018; 
Strausberg and Schreiber, 2003; Wang et al., 2016; Zhang et al., 2009). In infectious disease, small molecules, such as quorum sensing molecules, toxins and antibiotics, play a significant role in diagnosis and treatment (Donabedian, 2003; Hong-Geller and Micheva-Viteva, 2013; Pimbley and Patel, 1998). Mass spectrometry, nuclear magnetic resonance and liquid chromatography have been traditionally used as analytical methods for identification and quantification of small molecules or biomarkers (Dahal et al., 2011; Van, 2013; Zhou et al., 2017). These methods are generally complex, requiring skilled users, expensive instrumentation and often extensive sample preparation and analysis time. As a result, small molecule analysis has been largely restricted to specialised laboratories. There is a need for simple and low-cost technologies that can be implemented for rapid detection or realtime onsite or online monitoring of small molecules across varied applications and economic settings.

Label-free biosensors offer the potential to address the abovementioned need in small molecule detection (Nimse et al., 2016; Wang and Wang, 2008). Biosensors comprise a biorecognition element (or bioreceptor), such as an antibody, aptamer or molecular imprinted polymer, which binds specifically to the target molecule or marker, and a transducer that converts that binding into a recordable signal. Label-free biosensors are those that can transduce the binding event directly without the need for a secondary molecule (label), allowing relatively simpler and faster detection with less reagent use than labelled biosensors. Electrochemical and surface plasmon resonance (SPR) sensors are key examples of labelfree biosensors that allow rapid or realtime detection of small molecules with promising sensitivity and ability for miniaturisation (Harrad et al., 2018; Mitchell, 2010; Wang and Wang, 2008). However, non-specific binding remains one of the greatest challenges with biosensors that limits their adoption in practice (Nimse et al., 2016; Ronkainen et al., 2010). Moreover, the production cost, sensitivity to harsh conditions and limited shelf life of biological receptors, such as antibodies, and the cost and complexity of instrumentation, particularly of SPR biosensors, are other significant challenges limiting wide adoption of these biosensors (Nimse et al., 2016; Prabowo et al., 2018; Ronkainen et al., 2010).

Quartz crystal resonator (QCR), a thickness shear mode piezoelectric oscillator, is another important example of a platform for label-free biosensing (Vashist and Vashist, 2011). The changes in resonance frequency and dissipation (losses) of a QCR in response to binding of target biomolecules to specific receptors immobilised on the QCR are used to quantify the binding directly. QCRs are simple in configuration and entirely electronic, thus offering an excellent potential as a rapid, low-cost and portable biosensor. However, although QCRs have been successfully applied for relatively larger biomolecules, such as proteins and DNA, 
sensitive detection of small molecules has been challenging as the resulting changes in resonance frequency are low (Martínez-Rivas et al., 2010; Zheng et al., 2013). Gold nanoparticles have often been tagged to enhance the sensitivity of small molecule detection (Premaratne et al., 2017; Zheng et al., 2013). However, the use of nanoparticle tags adds complexity and cost, and not as suitable as a direct readout for rapid or realtime analysis.

\section{QCR using fixed frequency drive}

In our recent work, a novel method for determining shifts in resonance frequency and dissipation of a QCR using a fixed frequency drive was reported and named as 'fixed frequency drive' (FFD) method (Guha et al., 2019). Analytical expressions for shifts in resonance frequency and dissipation were presented for the first time using imaginary and real components of impedance respectively. The traditional methods for determining these parameters are impedance (or frequency sweep, FS) analysis or ring-down analysis. In impedance analysis, the QCR is driven by sweeping the frequency around its nominal resonance frequency and the admittance spectrum is fitted with a theoretical equivalent electrical circuit to estimate the resonance frequency and dissipation. In ring-down analysis, the QCR is excited with a narrow radio frequency pulse close to its nominal resonance frequency. The excitation is intermittently turned off and the transient response from the QCR is fitted with an exponentially decaying sinusoidal signal to estimate the resonance frequency and dissipation. In comparison, in the FFD method reported in our earlier work, these acoustic parameters are evaluated directly from each impedance data point using derived analytical expressions. Employing the FFD method with a QCR suggested the following advantages over the traditional methods that are vital for rapid and low cost online or onsite detection of small molecules.

First, there was no need for frequency synthesizers or fast analog-to-digital converters (ADCs) with a large dynamic range. Second, the time per measurement was significantly reduced as the acoustic parameters were evaluated analytically from each impedance data point and no time was spent for a sweep or ring-down or for fitting or averaging. These features allowed simpler and lower cost of instrumentation with high time resolution of measurement, potentially integrable on a single chip for the first time.

\section{Molecular imprinted polymer as receptor}

Molecular imprinted polymers (MIPs) are synthetic receptors that offer an attractive alternative to biological receptors, such as antibodies, by providing biosensors a long shelf life, stability and robustness in complex samples against harsh conditions. They also have a quick, simple 
and low-cost production process and a three-dimensional interfacial matrix that potentially offers a higher sensitivity against small molecules (Uludağ et al., 2007). It was shown recently that MIP nanoparticles (nanoMIPs) produced using an innovative solid-phase synthesis approach, which involves covalent immobilization of the template molecules onto the surface of a solid support (glass beads), provides a higher affinity of interaction between nanoMIPs and target molecules (Canfarotta et al., 2016).

In this piece of work, we explored a novel combination of a 14.3 MHz QCR, driven at a fixed frequency and amplitude and analysed using the above-mentioned FFD method, and a nanoMIP receptor, produced using solid-phase synthesis, to investigate the sensitivity and specificity of small molecule detection in a simple, rapid and direct (or label-free) format. We used an acyl-homoserine lactone ( $\mathrm{AHL}$ ), a class of quorum sensing molecules, as an exemplar of a small molecule. Bacterial cells communicate among each other employing certain chemical molecules as signals or auto inducers. Such a phenomenon is known as 'quorum sensing' (QS) (Kumari et al., 2008). Acyl-homoserine lactones (AHLs) are the most common class of quorum sensing molecules synthesized by gram-negative bacteria and often considered as a biomarker for infections caused by these bacteria. N-hexanoyl-L-homoserine lactone (C6-HSL), a type of AHL with molecular weight 199.25 Da, was used in this work. C6HSL mediated QS in opportunistic human pathogens, such as Serratia marcescens (Okano et al., 2012), has been found to be involved in nosocomial infections, including respiratory tract, urinary tract and wound infections (Annapoorani et al., 2012).

\section{AHL detection: State-of-the-art}

High Performance Liquid Chromatography (HPLC) (Poonguzhali et al., 2007), Liquid Chromatography coupled with Mass Spectrometry (LC-MS) (Middleton et al., 2002), High Performance Liquid Chromatography coupled with Mass Spectrometry (HPLC-MS) (Liu et al., 2017), Liquid Chromatography coupled with tandem Mass Spectrometry (LC-MS/MS) (Ortori et al., 2011), Gas Chromatography coupled with Mass Spectrometry (GC-MS) (Cataldi et al., 2007), Matrix Assisted Laser Desorption Ionisation Mass Spectrometry (MALDI-MS) (Kim et al., 2015), Nuclear Magnetic Resonance Spectroscopy (NMR) (Kumar et al., 2016) and Fourier Transform Infrared Spectroscopy (FTIR) (Taghadosi et al., 2015) have been explored for detection of AHLs from cell-free supernatants of gram negative bacterial cultures. Although, these detection techniques have achieved remarkable sensitivities in AHL quantification, they often require extensive sample pre-treatment and/or molecular derivatization steps as prerequisites for the analysis. Demand on operator time and skills along with high costs 
associated with massive instrumentation and assay performance restrict their use outside specialised laboratories as tools for detection of AHLs.

Label-free receptor-based biosensing methods with minimal sample processing steps have been explored to overcome some of the abovementioned limitations of conventional techniques. Surface plasmon resonance (SPR) (Wöllner et al., 2010) with monoclonal antibody has been employed for label-free realtime detection of AHLs. However, SPR faces numerous challenges in terms of miniaturization and cost reduction of instrumentation that is required for suitability of onsite use. In addition, there are limitations with cost of production of monoclonal antibodies and specificity, which affects reliability of detection. Electrochemical techniques, including differential pulse voltammetry (DPV) (Jiang et al., 2016) and cyclic voltammetry (CV) (Susmel and Comuzzi, 2017), have been explored for AHL detection using magnetic and electrochemically conductive MIPs respectively. Although the MIPs allow improvement in specificity, cost of production, robustness and long-term stability over antibodies, the need for electroactive biomolecular probe (Selvolini and Marrazza, 2017) and complexity in the set-up makes operation of miniaturised electrochemical biosensing techniques challenging (Mansouri, 2018). Thus, there lies an unmet need for label-free biosensing techniques that are low-cost, reliable and can be miniaturised for rapid, onsite or online detection of AHL.

\section{Aims}

As discussed earlier, QCR has the potential for delivering such a biosensor and is worth exploring. However, till date only one article has reported realtime detection of C6-HSL using a QCR (Okano et al., 2012). In that work, a $\beta$-cyclic oligosaccharide cyclodextrins ( $\beta$-CD) was used as the receptor for C6-HSL functionalised on a $27 \mathrm{MHz}$ QCR. The change in resonance frequency due to interaction between an aqueous solution of $\mathrm{C} 6-\mathrm{HSL}$ and $\beta-\mathrm{CD}$ receptor was measured using impedance or frequency sweep (FS) analysis. The minimum C6-HSL concentration detected in this work was $0.1 \mathrm{mM}$.

In this study, we employed a novel combination of a $14.3 \mathrm{MHz} Q \mathrm{QR}$, analysed using the analytical formula-based fixed frequency drive (FFD) method (Guha et al., 2019), and a nanoMIP receptor, produced in-house using solid-phase synthesis, to explore the feasibility of sensitive and specific label-free detection of C6-HSL. The lowest concentration of C6-HSL explored and detected in our work was $1 \mu \mathrm{M}$ (signal-to-noise ratio, SNR $>3$ ), which is approximately two orders of magnitude lower compared to the earlier work with $27 \mathrm{MHz} Q \mathrm{CR}$ using the traditional impedance analysis method and $\beta$-CD receptor (Okano et al., 2012). 


\section{Materials and Methods}

\subsection{Fixed Frequency Drive (FFD) method}

The QCR response was analysed using the Fixed Frequency Drive (FFD) method to determine the resonance frequency and dissipation (Guha et al., 2019). The analytical expressions of this method are based on the Butterworth Van-dyke (BVD) model, which gives the equivalent electrical circuit for a QCR. A QCR is a mechanical oscillator, commonly modelled using a spring, mass and damper (Figure 1a). The viscous damping $(c)$, mass $(\mathrm{m})$ and spring compliance $(1 / k)$ of the QCR oscillator are modelled by the resistance $\left(R_{m}\right)$, inductance $\left(L_{m}\right)$ and capacitance $\left(C_{m}\right)$ of the 'motional branch' of the BVD equivalent electrical circuit respectively (Figure 1b). The dielectric capacitance of the quartz and any holder capacitance are modelled by the shunt capacitance $\left(C_{0}\right)$.

a

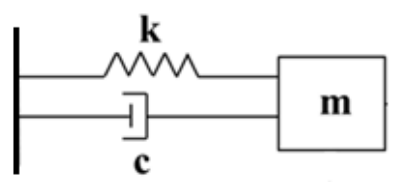

b

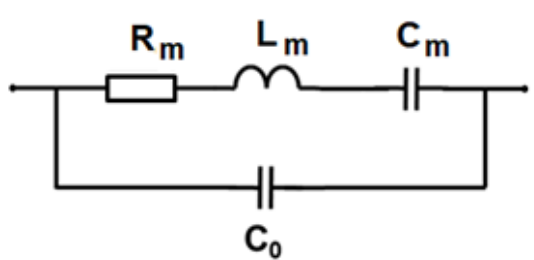

Figure 1. a. Spring mass damper model of a mechanical oscillator. b. Butterworth Van Dyke (BVD) model of a quartz crystal resonator (QCR)

In our earlier work, the reactance or the imaginary component of the motional impedance of the circuit, $X_{m}=\omega L_{m}-\frac{1}{\omega C_{m}}$, was alternatively expressed as follows using the characteristic impedance of the circuit, $\rho=\sqrt{L_{m} / C_{m}}$, and the resonance frequency, $\omega_{0}=1 / \sqrt{L_{m} C_{m}}$ (Guha et al., 2019).

$$
X_{m}=\rho\left(\frac{\omega}{\omega_{0}}-\frac{\omega_{0}}{\omega}\right)=\rho\left(\frac{f}{f_{0}}-\frac{f_{0}}{f}\right)
$$

The solution of Eq. 1 gave the following analytical expressions for resonance frequency (Eq. 2) and frequency offset, $\Delta f_{0}=f_{0}-f$, (Eq. 3). The detailed derivation and justification were provided in the paper (Guha et al., 2019).

$$
f_{0}=\frac{f}{2 \rho}\left(-X_{m}+\sqrt{X_{m}^{2}+4 \rho^{2}}\right)
$$




$$
\Delta f_{0}=\frac{f}{2 \rho}\left(-X_{m}+\sqrt{X_{m}^{2}+4 \rho^{2}}\right) \approx-f \frac{X_{m}}{2 \rho}\left(1-\frac{1}{2} \frac{X_{m}}{2 \rho}\right)
$$

The drive frequency, $f$, is known and kept constant. It was justified that the characteristic impedance, $\rho$, could be treated as a constant for a batch of crystals and pre-determined from the manufacturer or impedance analysis. Hence, the resonance frequency can be determined from the measured reactance data, $X_{m}$.

\subsection{Synthesis of nanoMIPs using solid-phase molecular imprinting}

MIP nanoparticles (NanoMIPs) were synthesized using solid-phase molecular imprinting technique (Canfarotta et al., 2016), which involves covalent immobilisation of template molecule ((S)-(-)- $\alpha$-Amino- $\gamma$-butyrolactone hydrobromide) on the surface of glass beads (53$106 \mu \mathrm{m}$ ) followed by polymerisation and subsequent affinity separation step involving the washing of beads for removal of poor binders and unproductive polymers. Polymerisation was conducted under the exposure of ultra-violet (UV) light and in presence of methacrylic acid monomers, cross-linker, including ethylene glycol dimethacrylate (EGDMA) and trimethylolpropane trimethacrylate (TRIM), and organic solvent, including acetonitrile. The chemical reaction flow diagram is presented in Figure 2. Details of reagents and protocol are given below.

Reagents. Methacrylic acid (MAA), ethylene glycol dimethacrylate (EGDMA), phosphate buffered saline (PBS), pentaerythritol-tetrakis-(3-mercaptopropionate) , 3aminopropyltrimethyloxysilane (APTMS), trimethylolpropane trimethacrylate (TRIM), dodecanedioic acid, (S)-(-)-a-Amino-y-butyrolactone hydrobromide, sodium carbonate $\left(\mathrm{Na}_{2} \mathrm{CO}_{3}\right)$, sodium chloride $(\mathrm{NaCl}), \mathrm{N}$-hydroxysuccinimide (NHS), Cystamine, methanol, acetone, toluene and isopropanol were purchased from Sigma Aldrich, UK. PBS comprised of $8.1 \mathrm{mM} \mathrm{Na}_{2} \mathrm{HPO}_{4}, 1.1 \mathrm{mM} \mathrm{KH}_{2} \mathrm{PO}_{4}, 1 \mathrm{mM} \mathrm{MgCl}, 2.7 \mathrm{mM} \mathrm{KCl}$, and $138 \mathrm{mM} \mathrm{NaCl}$ with a pH of 7.4. Deionised (DI) water was obtained from Ultrapure Milli-Q water (Millipore, UK). 1-ethyl3-(3-dimethylaminopropyl)-carbodiimide hydrochloride (EDC), Sodium hydroxide $(\mathrm{NaOH})$, Dimethylformamide (DMF), ethanol and dry acetonitrile (ACN) were purchased from Fisher Scientific (UK). The MES buffer (2-[morpholino] ethanesulfonic acid) was obtained from Thermo Scientific (UK). N-hexanoyl-L-Homoserine lactone (C6-HSL) was obtained from Cambridge Bioscience. N, N'-diethyldithiocarbamic acid benzyl ester (Iniferter) were obtained from TCl Europe (Belgium). Glass beads (Spheriglass $₫ 2429$ ) with diameters varying from 53 $-106 \mu \mathrm{m}$ were purchased from Blagden Chemicals, UK. Centrifugal filter units (Amicon Centriplus ${ }^{\circledR}$ ) with $30 \mathrm{kDa}$ molecular weight cut-off were procured from Millipore, UK. 
Protocol for preparation of anti- $\mathrm{C}_{6}-\mathrm{HSL}$ glass beads. Glass beads were first shaken with ceramic beads on $45 \mu \mathrm{m}$ sieves for three to four hours using a Vibratory Sieve Shaker AS 200 basic (Retsch, UK) in order to abrade the surface coating and expose a fresh surface. After recovery from the sieve, the surface of the glass beads was activated by boiling with $2 \mathrm{M}$ $\mathrm{NaOH}$ for 15 min, followed by rinsing with deionised (DI) water and acetone, and then drying at $80{ }^{\circ} \mathrm{C}$. The dry beads were incubated overnight in $2 \% \mathrm{v} / \mathrm{v}$ APTMS/toluene solution to introduce surface amino groups.

EDC/NHS chemistry was used to crosslink carboxylic groups with primary amines. Briefly, in order to get an activation buffer $(\sim 100 \mathrm{~mL}), 2.172 \mathrm{~g}(11.12 \mathrm{mmol})$ of MES was mixed with $2.922 \mathrm{~g}(49.99 \mathrm{mmol})$ of $\mathrm{NaCl}$ in distilled water and its $\mathrm{pH}$ was adjusted to 6.0 by adding few drops of $\mathrm{NaOH}$ (1 or $2 \mathrm{M}$ ). An activation mixture was then prepared in a $50 \mathrm{~mL}$ volumetric flask by adding $0.125 \mathrm{~g}(5.43 \mathrm{mmol})$ of dodecanedioic acid to $20 \mathrm{~mL}$ of activation buffer. After that, $2.0 \mathrm{~g}(0.01 \mathrm{mmol}) \mathrm{EDC}$ and $0.420 \mathrm{~g}(3.65 \mathrm{mmol}) \mathrm{NHS}$ were dissolved into $10 \mathrm{~mL}$ of fresh activation buffer and then the resulting solution was added to the dodecanedioic acid solution and mixed by Vortex Shaker. The glass beads functionalised with APTMS were incubated for 20 minutes in the prepared solution. After that, the beads were washed with $50 \%$ DMF in water, activation buffer and acetone. The immobilisation of the template ((S)-(-)- $\alpha-A m i n o-\gamma-$ butyrolactone hydrobromide) on the surface of the beads was performed by incubating the glass beads functionalised with the activated dodecanedioic acid in an aqueous solution ( $~ 50$ $\mathrm{mL})$ containing $0.07908 \mathrm{~g}(4.43 \mathrm{mmol})$ of the template and $0.046 \mathrm{~g}(4.344 \mathrm{mmol})$ of sodium carbonate. The immobilisation was carried out for 2 hours. The beads were washed with ultrapure water. Finally, the glass beads were dried under vacuum and stored in the fridge until next use. The actual mass of glass beads used for templating in the MIP was $40 \mathrm{~g}$. 

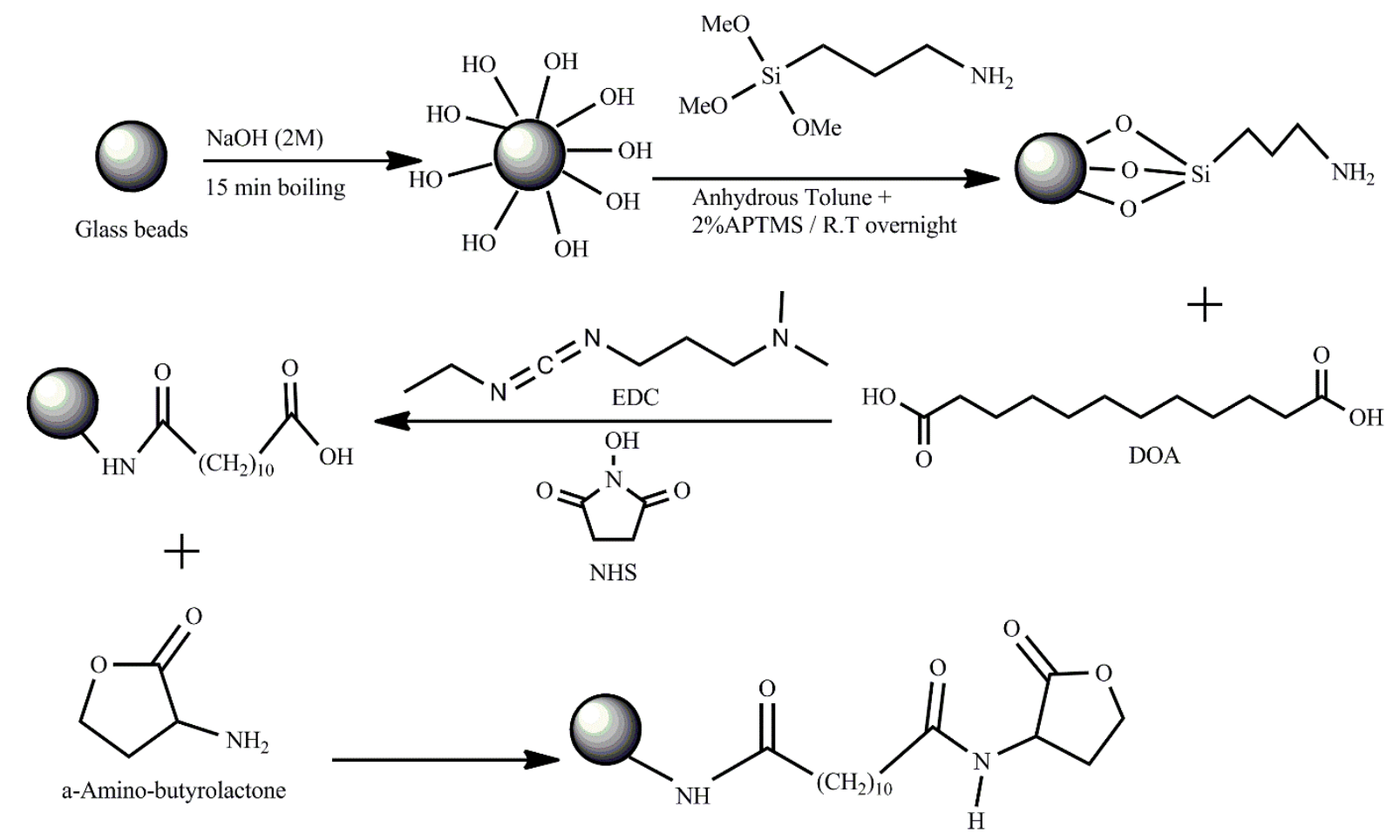

Figure 2. Flow chart describing the synthesis of templating molecules

Protocol for polymerisation and anti-C6-HSL nanoMIP synthesis. A polymerisation mixture was prepared in a $20 \mathrm{~mL}$ vial by mixing $0.3 \mathrm{~g}(0.61 \mathrm{mmol})$ pentaerythritol-tetrakis-(3mercaptopropionate) as a Chain Transfer Agent (CTA), $1.255 \mathrm{~g} \mathrm{(5.2} \mathrm{mmol)} \mathrm{Iniferter,} 4.8 \mathrm{~g}$ (55.78 mmol) MAA as the backbone monomer, $5.4 \mathrm{~g}$ (27.2 mmol) EGDMA, $5.4 \mathrm{~g} \mathrm{(15.95} \mathrm{mmol)}$ TRIM acting as cross-linkers and $17.55 \mathrm{~g}(247.5 \mathrm{mmol})$ of $A C N$. The solution was mixed well and filtered through a $0.45 \mu \mathrm{m}$ Teflon filter to remove any insoluble compound into a new vial covered with aluminium foil. The derivatised glass beads were incubated in a beaker sealed with a lid. The beaker was then placed for 10 minutes in a vacuum oven to remove the oxygen. The polymerisation mixture was added to the beaker and sandwiched between two UV light sources (Philips model HB/171/A, each fitted with $4 \times 15 \mathrm{~W}$ lamps) for $90 \mathrm{sec}$. The anti-AHL glass beads and polymerisation mixture were poured into a polyethylene solid-phase extraction cartridge fitted with a frit (20 $\mu \mathrm{m}$ porosity) in order to separate glass beads (with the nanoparticles attached) from the other components. Two different washing steps were performed. Firstly, eight equivalents of cold $\mathrm{ACN}\left(0^{\circ} \mathrm{C}\right)$ were used to remove unreacted polymer and low affinity particles. This was followed by five washes with hot $A C N\left(60-65^{\circ} \mathrm{C}\right)$ to elute high affinity nanoparticles. The final total volume of the high affinity anti- $\mathrm{C}_{6}-\mathrm{HSL}$ MIP nanoparticles was about $100 \mathrm{ml}$. The concentration of the MIP nanoparticles was $0.21 \mathrm{mg} / \mathrm{ml}$ in $100 \mathrm{ml}$ of acetonitrile. The MIP nanoparticles were kept refrigerated in acetonitrile solvent. 
Protocol for preparation of $\mathrm{C}_{6}-\mathrm{HSL}$ stock solution. $5.9 \mathrm{mg}$ of $\mathrm{C} 6-\mathrm{HSL}$ with a molecular weight of 199.25 Da was added in $3 \mathrm{ml}$ PBS to prepare $0.1 \mathrm{M}$ C6-HSL stock solutions.

\subsection{QCR sensor preparation and assembly}

Immobilisation of QCR with anti-C6-HSL nanoMIP. 14.3 MHz AT-cut thickness-shear mode quartz crystal resonators (diameter: $8.3 \mathrm{~mm}$; thickness: $115 \mu \mathrm{m}$; from LapTech Precision Inc., Bowmanville, Ontario, Canada) were cleaned using ultrasonication in acetone (5 min) and isopropanol (10 $\mathrm{min}$ ) and then treated in argon plasma (45 seconds) using a Harrick Plasma Cleaner. The cleaned QCRs were incubated overnight in an ethanolic solution of cystamine with a concentration of $0.2 \mathrm{mg} / \mathrm{ml}$. The anti-C6-HSL MIP nanoparticles solution was transferred from the acetonitrile to water by using centrifugal filter units. The QCRs, after overnight incubation with cystamine, were washed thoroughly in ethanol. An activated antiC6-HSL MIP nanoparticles solution was prepared by adding EDC alone to the anti-C6-HSL MIP nanoparticles solution. The activation with EDC was used more than one time with new MIPs. The cystamine-functionalised QCRs were incubated for 20 minutes in activated antiC6-HSL MIP nanoparticles solution to produce the QCR sensors.

Sensor assembly. A QCR sensor was assembled between a printed circuit board (PCB), which provided the electrical connection, and a CNC-milled acrylic microfluidic cartridge, which allowed the liquid flow. A UPS VI classified EPDM O-ring (Trelleborg AB, Sweden), fitted underneath the cartridge, helped contain the liquid $(\sim 10 \mu \mathrm{L})$ on top of the QCR sensor. The steps of the assembly are illustrated in Figure 3a. A pair of stationary clips (not shown) was used to hold the assembly together. The quality factor values of all assembled QCR sensors in this work were $\sim 1900$ in liquid, suggesting negligible damping by the O-ring. 


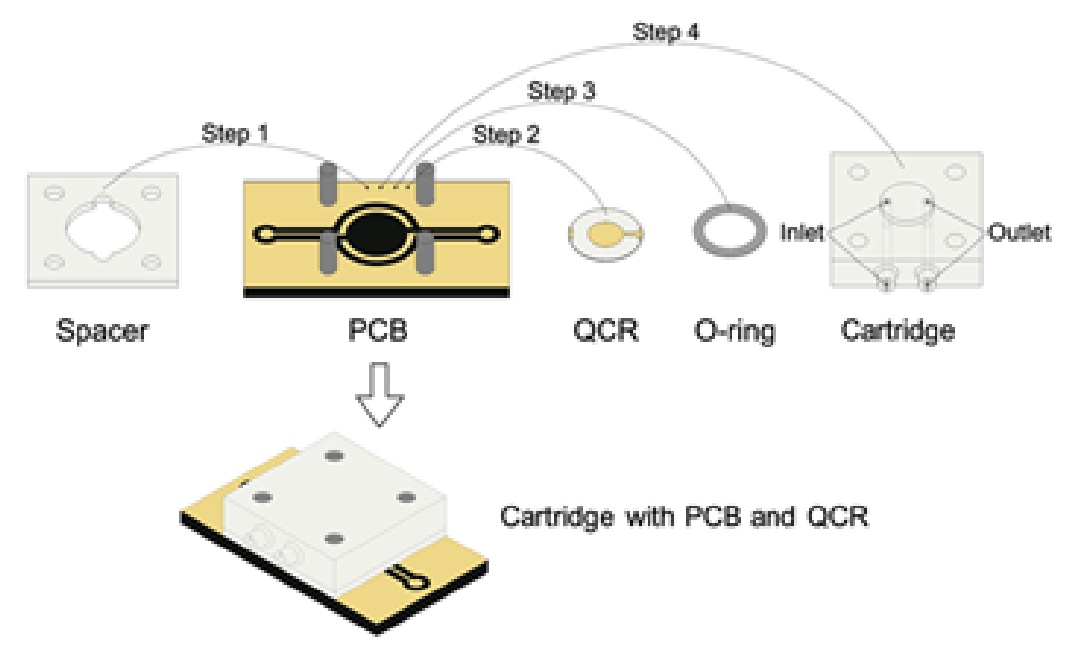

b

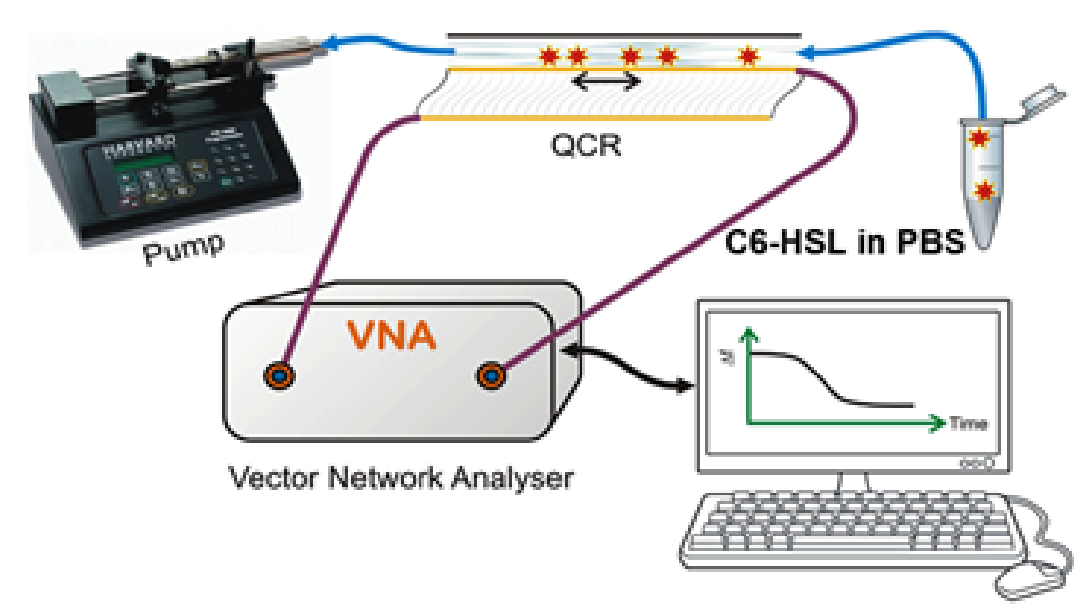

Figure 3. a. The sensor assembly. b. Schematic of the experimental set-up

\subsection{QCR experimental set-up}

A QCR sensor was driven using a network analyser, built in-house, which allowed both varying frequency and fixed frequency sweeps (Guha et al., 2019). The recording of data was controlled from a PC using a graphical user interface, custom-build for the network analyser. The data was analysed using Mathematica 10. A syringe pump (Harvard Apparatus) was used for controlled and measured delivery of sample onto the QCR sensor. A schematic of the setup is shown in Figure $\mathbf{3 b}$.

\subsection{Benchmarking with BIAcore SPR system}

For the purpose of benchmarking, Surface Plasmon Resonance (SPR) experiments were carried out with the same pair of C6-HSL and anti-C6-HSL nanoMIP. A BIAcore SPR system was employed. 
Preparation of BIAcore gold chips. Au-coated chips (SIA Kit Au, BIAcore) were treated with hydrogen plasma to clean the surface of contaminants. After that, the gold chips were placed in $1 \mathrm{mg} / \mathrm{mL}$ ethanolic solution containing cystamine for 24 hours. Then, the chips were washed in succession by ethanol and DI water, followed by nitrogen drying. This process was undertaken to introduce amino groups onto the surface of the chips. EDC/NHS chemistry was then used to bind the nanoMIPs onto the surface of the chips. The nanoMIP sample was sonicated for 3 min prior to use.

Preparation of samples for BIAcore experiments. Serial dilutions of template were made using sample buffer (0.01 M PBS). $500 \mu \mathrm{L}$ of each concentration were prepared in Eppendorf tubes. The decision to increase or decrease the concentration of the template was determined by the BIAcore experiment itself. Examples of the dilutions used were: 1:1, 1:2, 1:4, 1:8, 1:16, $1: 32,1: 64,1: 128$ and $1: 256$.

Running the BIAcore experiment. The BIAcore is an automated system that requires the sample to be placed into a given compartment. The rest of the experiment is controlled by the BIAcore software. The flow rate was $15 \mu \mathrm{l} / \mathrm{min}$. For the experiment, $100 \mu \mathrm{L}$ of C6-HSL was injected. KINJECT mode was used, which has fixed times between injections in order to monitor dissociation. Injections started with the most dilute (low concentration) to least dilute (high concentration). The evaluation of the results was carried out using the BIA evaluation software.

\section{Results}

\subsection{Detection of C6-HSL}

Experiments for realtime detection of C6-HSL in phosphate buffer saline (PBS) were conducted using 14.3 MHz QCRs functionalised with anti-C6-HSL nanoMIP. Shifts in resonance frequency and dissipation were determined using both the novel fixed frequency drive (FFD) method and the traditional impedance analysis or frequency sweep (FS) method and compared quantitatively. Sensitivity of detection was explored with a range of concentration of C6-HSL $(1 \mu \mathrm{M}-50 \mu \mathrm{M})$ and compared with SPR, which is a gold standard label-free detection method in the laboratory. Specificity of detection was explored using dopamine solution $(50 \mu \mathrm{M})$ as a negative control.

Two types of scans were taken every 2 min during the baseline and the binding phases: frequency sweep around $14.3 \mathrm{MHz}(0.1 \mathrm{sec}, 0.52 \mathrm{~V})$ and fixed frequency and amplitude scan near $14.3 \mathrm{MHz}(0.1 \mathrm{sec}, 0.47 \mathrm{~V})$. The parameters of the equivalent circuit $\left(R_{m}, L_{m}, C_{m}\right.$ and $C_{0}$ in Figure 1b) were estimated by fitting the electrical impedance recorded from the frequency 
sweep with the BVD model. This allowed the determination of characteristic impedance, $\rho=$ $\sqrt{L_{m} / C_{m}}$, and resonance frequency, $\omega_{0}=1 / \sqrt{L_{m} C_{m}}$, according to the impedance analysis (FS) method. The resonance frequency according to the fixed frequency drive (FFD) method was determined from the fixed frequency and amplitude scan using Eq. 3. The motional reactance, $X_{m}=\operatorname{Im}\left(Z_{m}\right)$, required for Eq. 3 was obtained from the equivalent circuit as $Z_{m}=$ $\left(1 / Z-j \omega C_{0}\right)^{-1}$, where $Z$ is the equivalent impedance of the motional and shunt arms that is experimentally recorded from the fixed frequency and amplitude scan. The characteristic impedance, $\rho$, and shunt capacitance, $C_{0}$, obtained from the frequency sweep fit were assumed to be constant for all QCR crystals in a batch and used in Eq. 3.

A baseline was established in PBS under flow (at $40 \mu \mathrm{L} / \mathrm{min}$ ) by taking both frequency sweep and FFD scans every 2 min over $30 \mathrm{~min}$. These scans were continued to be taken during the C6-HSL-nanoMIP binding phase when C6-HSL solution in PBS was injected over 30 min (at $40 \mu \mathrm{L} / \mathrm{min})$. A range of concentrations of C6-HSL solution was explored (1 $\mu \mathrm{M}-50 \mu \mathrm{M})$. Separate QCR sensors were employed for each concentration of C6-HSL. The resonance frequency shifts (relative to the first baseline point) were determined over the baseline and C6-HSL-nanoMIP binding phases using the impedance analysis (FS) and FFD methods (Figure 4a). The baseline showed satisfactory stability with $<10 \mathrm{~Hz}$ drift over $30 \mathrm{~min}$ and standard deviation noise of $\sigma=0.63 \mathrm{~Hz}$ and $\sigma=0.46 \mathrm{~Hz}$ for impedance analysis and FFD methods respectively. The baseline and growth curves, showing progress of binding, were always in good agreement between the two methods and for all concentrations of C6-HSL. The resonance frequency shift measured using FFD after $30 \mathrm{~min}$ of C6-HSL injection agreed within $2 \%-5 \%$ of the same measured using frequency sweep for $2.5 \mu \mathrm{M}-50 \mu \mathrm{M}$ of C6-HSL concentration (Figure $4 \mathrm{~b})$. The agreement was relatively poorer $(\sim 20 \%)$ at $1 \mu \mathrm{M}$ due to the low absolute value of the shift $(\sim 25 \mathrm{~Hz})$ at this concentration. Figure $4 \mathbf{b}$ also demonstrates quantitative correlation of frequency shift with C6-HSL concentration. 

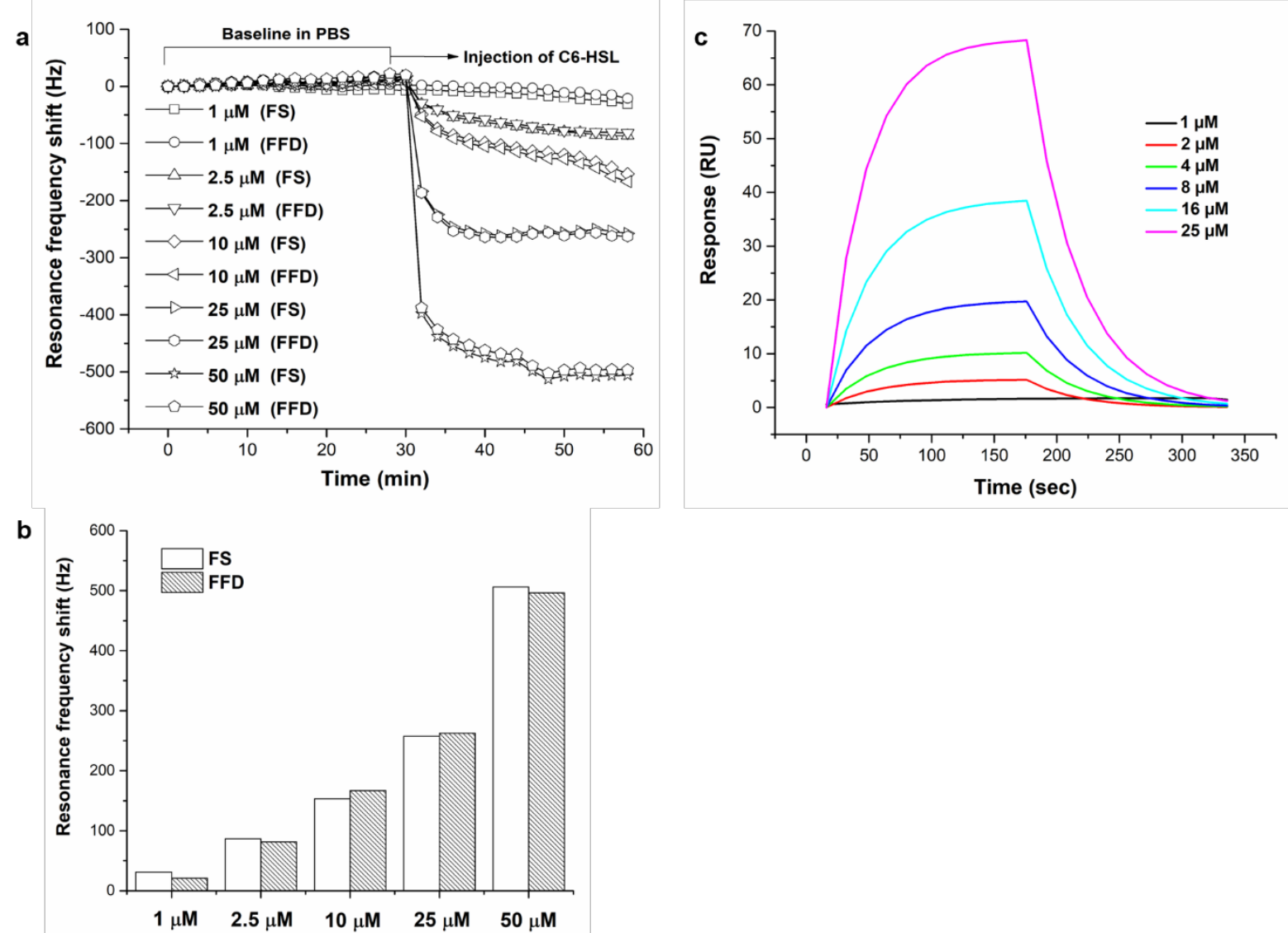

Figure 4. a. Resonance frequency shift over baseline and C6-HSL-nanoMIP binding phases b. Resonance frequency shift after $30 \mathrm{~min}$ of $\mathrm{C} 6-\mathrm{HSL}$ injection for various C6-HSL concentrations using frequency sweep and FFD methods. c. A representative SPR response (change in refractive index) from the association and dissociation of C6-HSL with anti-C6-HSL nanoMIP.

\subsection{Sensitivity}

The minimum C6-HSL concentration explored and detected in this work using QCR and nanoMIP was $1 \mu \mathrm{M}$ (sample volume $800 \mu \mathrm{L}$ ), which is two orders of magnitude lower than that reported in the only other C6-HSL detection study in the literature using a QCR, where $\beta$-cyclic oligosaccharide cyclodextrins ( $\beta-C D)$ was used as the receptor (Okano et al., 2012). The reported QCR-nanoMIP sensor demonstrated a significant improvement in sensitivity above the earlier work despite the fact that a $27 \mathrm{MHz}$ QCR was used in the earlier work, which according to Sauerbrey equation (Sauerbrey, 1959), has a higher mass sensitivity per unit area than a 14.3 MHz QCR used in this work (Garcia et al., 2011). To benchmark the performance of the QCR sensor with a laboratory gold standard for label-free detection, experiments were also carried out with a BIAcore SPR instrument as described in Section 2.5. The change in refractive index was measured by the instrument in real time as binding of C6HSL and anti-C6-HSL nanoMIP progressed (a representative dataset shown in Figure 4c). 
The lowest concentration of C6-HSL detected (in multiple experiments) in this set-up was $2 \mu \mathrm{M}$, whereas the lowest concentration detected with our QCR-nanoMIP set-up was $1 \mu \mathrm{M}$. So even with the current unoptimized QCR set-up, the limit of detection was comparable with SPR, which is a significantly more complex and expensive instrument.

With our current QCR-nanoMIP set-up, the shift in resonance frequency measured for $1 \mu \mathrm{M}$ C6-HSL was $\sim 25 \mathrm{~Hz}$ with an Allan Deviation noise of $1.83 \mathrm{~Hz}$ using the FFD method. Determination of limit of detection was not one of the aims of this work. However, the abovementioned signal-to-noise ratio suggests the potential for a lower limit of detection (LoD) than $1 \mu \mathrm{M}$. The exploration of lower C6-HSL concentrations was not attempted in this work as a frequency drift of 3-10 Hz over 30 min was observed in this set-up. The installation of a temperature stabiliser is expected to reduce this drift. Once this is done, it will then be worthwhile exploring lower C6-HSL concentrations and determining the LoD and binding kinetics of the current set-up with measurement of replicates.

\subsection{Specificity}

Dopamine was chosen as the negative control for the specificity study as it has similar molecular weight (153.18 Da) compared with C6-HSL (199.2 Da). Like the experiments with C6-HSL, a baseline was recorded by flowing PBS over the QCR sensor (functionalised with anti-C6-HSL nanoMIP) for $30 \mathrm{~min}$ at $40 \mu \mathrm{L} / \mathrm{min}$. $50 \mu \mathrm{M}$ Dopamine solution was then injected over the sensor for the next $30 \mathrm{~min}$ at the same flow rate. Frequency sweep and fixed frequency/amplitude scans were taken every $2 \mathrm{~min}$ in the baseline and dopamine injection phases, and frequency shifts compared to the baseline were recorded similar to that done for C6-HSL binding experiments. The conductance graphs from the frequency sweeps are shown in Figure 5. The frequency shift measurements from the FFD method agreed well with that from impedance analysis (FS) over the baseline and injection phases for C6-HSL and Dopamine flow experiments at all times (Figure 6a). The final frequency shifts after $30 \mathrm{~min}$ flow were comparable too (Figure 6b). This once again validated the quantitative agreement of the FFD and the impedance analysis methods. Negligible shift ( $-9 \mathrm{~Hz}$ according to FFD method) was observed after 30 min of Dopamine injection compared to the shift in the case of C6-HSL ( $-495 \mathrm{~Hz}$ according to FFD method). The significantly lower shift ( $\sim 56$ fold) obtained with negative control of the same concentration suggested a high specificity of detection.

As a negative control, another set of experiments with identical measurements was conducted. Here PBS was flowed (at $40 \mu \mathrm{L} / \mathrm{min}$ ) continuously over the same QCR sensor for $60 \mathrm{~min}$. Negligible shift in resonance frequency $(<10 \mathrm{~Hz})$ was observed in the last 30 min (Figure 5a), which can be attributed to the drift of the QCR. This drift is relatively low for a QCR that is 
functionalised with bioreceptors, suggesting a good quality of surface functionalisation. The drift can be further reduced with the installation of a temperature stabiliser as mentioned in Section 3.2.

Reaction of QCR sensor with PBS

b Reaction of QCR sensor with C6-HSL

c Reaction of QCR sensor with Dopamine
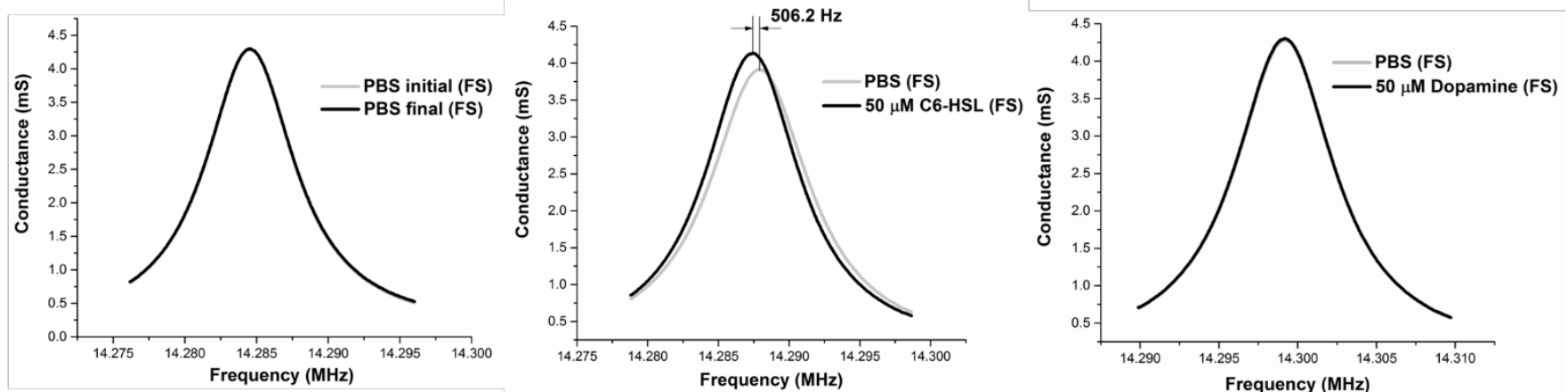

Figure 5. a. Conductance graphs of the QCR sensor in PBS for two frequency sweeps with 30 min interval. Negligible shift in the peak of conductance graph (i.e. resonance frequency) observed. b. Conductance graphs of the QCR sensor in PBS and after flowing $50 \mu \mathrm{M}$ C6-HSL solution for $30 \mathrm{~min}$. Shift in resonance frequency of $\sim 506 \mathrm{~Hz}$ observed. c. Negligible shift in resonance frequency observed after flowing $50 \mu \mathrm{M}$ Dopamine solution over the same QCR sensor for $30 \mathrm{~min}$.
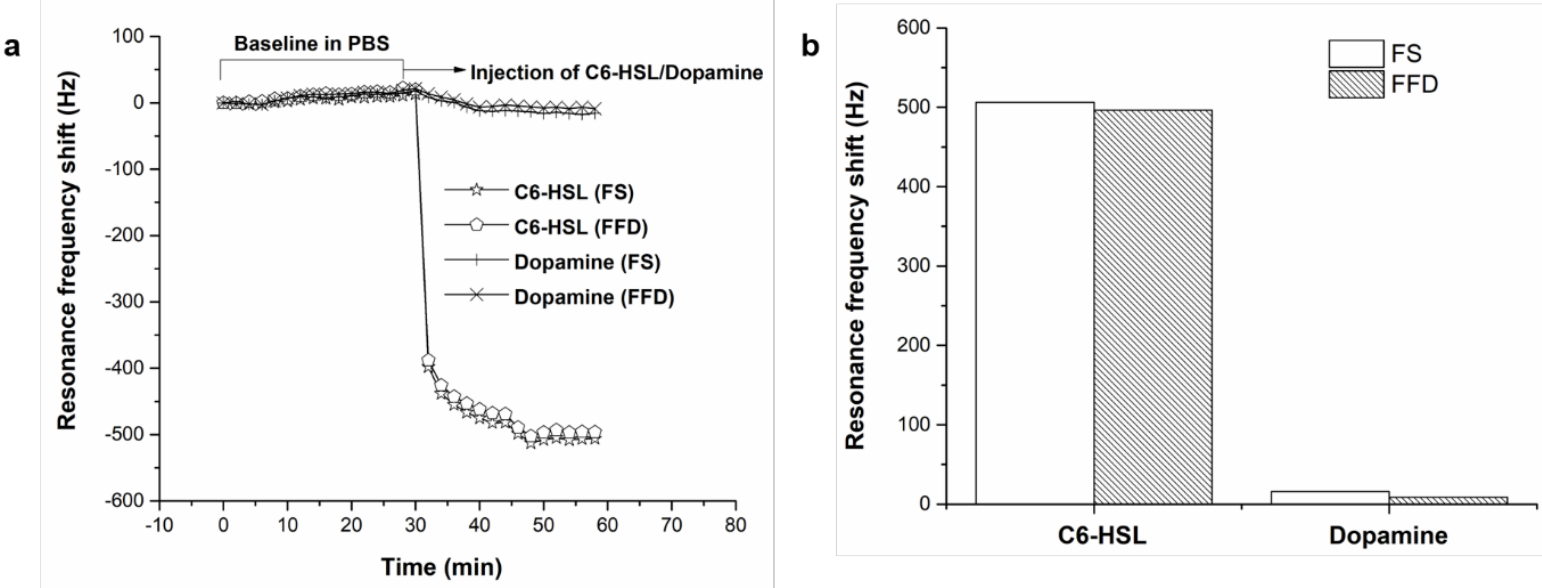

Figure 6. a. Resonance frequency shifts due to binding of $50 \mathrm{mM} \mathrm{C6-HSL}$ and $50 \mathrm{mM}$ Dopamine on functionalised QCRs using frequency sweep and FFD methods with 2 min measurement interval b. Resonance frequency shift $30 \mathrm{~min}$ after $50 \mathrm{mM} \mathrm{C6-HSL}$ and 
Dopamine injections c. Variation in the resonance frequency shifts after 30 min for FFD (50 $\mathrm{mM}$ C6-HSL and $50 \mathrm{mM}$ Dopamine) with respect to frequency sweep (FS) method.

The control experiments suggested high specificity of detection of C6-HSL using QCR functionalised with anti-C6-HSL nanoMIP receptor. A 56-fold greater shift was observed for C6-HSL compared to that from a similar concentration of the negative control (Dopamine). This was a notable advancement beyond the state-of-the-art in specificity of small molecule detection using QCR. Ochratoxin A (OTA, MW 404 Da) was reported to be detected using $5 \mathrm{MHz}$ QCR functionalised with antibodies (Pirinçci et al., 2018). The frequency shift obtained with OTA (specific binding) was $~ 10$-fold of that obtained with BSA (non-specific binding), which is a typical figure for differentiation between specific and non-specific binding with antibody-based assays.

\section{Discussion}

We anticipate that the use of nanoMIP as the receptor is one of the key reasons behind the high sensitivity and specificity observed in this study. Through molecular modelling using Sybyl, Piletska et al confirmed that methacrylic acid (MAA) monomers have strong interactions with AHLs (Piletska et al., 2011, 2010). Particularly, the keto-functional group of AHLs is suitable for binding to MAA (Weiss et al., 2003). The molecularly imprinted polymer nanoparticles (nanoMIPs) used in this work contained MAA monomers. Besides the nanoMIP, the low operating noise $(\sim 1 \mu \mathrm{V} / \sqrt{\mathrm{Hz}})$ of the network analyser instrument also played a role in achieving the detection of a low concentration $(1 \mu \mathrm{M})$ of a small molecule (C6-HSL, $199 \mathrm{Da})$ using a QCR. Since the instrument noise was far below the noise from the biomolecular surface, it allowed focusing on improving the assay for lower noise from the surface. A frequency shift of $\sim 25 \mathrm{~Hz}$ was recorded for the binding of $1 \mu \mathrm{M}$ C6-HSL over 30 min with an Allan Deviation noise of $1.83 \mathrm{~Hz}$ at a sampling rate of 1 frequency datapoint every 2 minutes. This signal-tonoise ratio from this proof of principle study already suggests the potential for a limit of detection lower than $1 \mu \mathrm{M}$. Applying the FFD method with a higher data sampling rate and averaging, which it is uniquely capable of, can further lower the potential limit of detection (LoD). However, a drift of $\sim 10 \mathrm{~Hz}$ over $30 \mathrm{~min}$ was found as a limitation. Installation of a temperature stabiliser to cancel or reduce the drift should allow the exploration of C6-HSL concentrations lower than $1 \mu \mathrm{M}$ and determination of LoD of the current set-up with measurement of replicates. It will also be worthwhile optimising the QCR-nanoMIP set-up for greater sensitivity. The sensitivity per unit area grows with fundamental resonance frequency according to Sauerbrey equation (Garcia et al., 2011) albeit at the cost of lower quality factor (i.e. higher dissipation). Hence, an optimal drive frequency needs to be established. Mesa crystals of $50 \mathrm{MHz}$ or $100 \mathrm{MHz}$ frequency are worth exploring in this regard (March et al., 
2015). Investigating a range of nanoMIP concentration on the QCR is also important to optimise the sensitivity. The influence of electrode material on the mass sensitivity of a QCR is known and is getting increasingly clearer through recently reported studies (Huang et al., 2019). Optimising the electrode material can further enhance the sensitivity of the reported QCR-nanoMIP sensor. The nonuniformity of QCR's mass sensitivity across its diameter often limits its quantitative reproducibility and thereby the limit of detection. Improvement in uniformity of mass sensitivity across the QCR diameter, for example through using a ring electrode with optimised inner and outer radii, will be important for improved quantitative accuracy and limit of detection of this QCR-nanoMIP sensor (Huang et al., 2018).

\section{Conclusion}

Combining nanomolecular imprinted polymer (nanoMIP) with QCR actuated and analysed with fixed frequency drive (FFD) method demonstrated sensitive and specific label-free detection of N-hexanoyl-L-homoserine lactone (C6-HSL), a 199 Da quorum sensing molecule. The QCR-nanoMIP sensor operating on FFD method offers the potential for a simple, scalable and affordable technology for rapid/realtime detection or onsite/online monitoring of small molecules. MIPs are quick and cheap to produce, stable in complex sample and harsh conditions, and allow sensor regeneration and longer shelf life, facilitating practical implementation. MIPs also offer a 3D interfacial matrix with higher sensitivity against small molecules. In addition, nanoMIPs produced using solid-phase synthesis, as in this work, provide higher affinity leading to the demonstrated improvement in sensitivity and specificity the fundamental limiting challenges in small molecule detection. Likewise, while QCR offers the advantages of a label-free sensor, the analytical formula-based fixed frequency drive (FFD) method to actuate and read the QCR significantly improves the simplicity in instrumentation. With no need for frequency synthesizers or fast ADCs, FFD can potentially allow the first "QCR-on-chip", which can bring a paradigm shift in scalability, accessibility and affordability of small molecule detection. The formula-based method allowing analog/digital averaging of linear impedance signal can potentially offer the fastest possible measurements with the lowest possible noise with frequency drift minimised using a temperature stabiliser. It will be worth exploring the feasibility of large-scale multiplexability of small molecule detection at low cost by utilising the unique possibility with FFD for calibration at a single frequency to eliminate electromagnetic coupling, which is currently expensive to eliminate.

\section{Acknowledgements}

The authors acknowledge the contributions of Dr Alexander Zhukov of the University of Cambridge for development of the graphical user interface of the vector network analyser instrument, the EU projects RAPP-ID (FP7-JTI 115153) and Norosensor (FP7-NMP 604244), 
and the EPSRC Bridging the Gap in Antimicrobial Resistance grant (EP/M027341/1) for covering the cost of instrument development and consumables, and Wolfson School for supporting the PhD studentship of AG.

\section{References}

Annapoorani, A., Jabbar, A.K.K.A., Musthafa, S.K.S., Pandian, S.K., Ravi, A.V., 2012. Inhibition of Quorum Sensing Mediated Virulence Factors Production in Urinary Pathogen Serratia marcescens PS1 by Marine Sponges. Indian J. Microbiol. 52, 160 166. https://doi.org/10.1007/s12088-012-0272-0

Canfarotta, F., Poma, A., Guerreiro, A., Piletsky, S., 2016. Solid-phase synthesis of molecularly imprinted nanoparticles. Nat. Protoc. 11, 443-455. https://doi.org/10.1038/nprot.2016.030

Cataldi, T.R.I., Bianco, G., Palazzo, L., Quaranta, V., 2007. Occurrence of N-acyl-Ihomoserine lactones in extracts of some Gram-negative bacteria evaluated by gas chromatography-mass spectrometry. Anal. Biochem. 361, 226-235. https://doi.org/10.1016/j.ab.2006.11.037

Dahal, U.P., Jones, J.P., Davis, J.A., Rock, D.A., 2011. Small molecule quantification by liquid chromatography-mass spectrometry for metabolites of drugs and drug candidates. Drug Metab. Dispos. 39, 2355-2360. https://doi.org/10.1124/dmd.111.040865

Donabedian, H., 2003. Quorum sensing and its relevance to infectious diseases. J. Infect. 46, 207-214. https://doi.org/10.1053/jinf.2002.1120

Garcia, J. V, Jimenez, Y., March, C., Montoya, A., Arnau, A., 2011. QCM technology in biosensors. Biosens. - Emerg. Mater. Appl. 153-178. https://doi.org/10.5772/17991

Gooding, J.J., 2006. Biosensor technology for detecting biological warfare agents: Recent progress and future trends. Anal. Chim. Acta 559, 137-151. https://doi.org/10.1016/j.aca.2005.12.020

Grams, M.E., Shafi, T., Rhee, E.P., 2018. Metabolomics research in chronic kidney disease, in: Journal of the American Society of Nephrology. pp. 1588-1590. https://doi.org/10.1681/ASN.2018030256

Guha, A., Sandström, N., Ostanin, V.P., Wijngaart, W. van der, Klenerman, D., Ghosh, S.K., 2019. Simple and ultrafast resonance frequency and dissipation shift measurements using a fixed frequency drive. Sensors Actuators B Chem. 281, 960-970. https://doi.org/doi.org/10.1016/j.snb.2018.11.052

Harrad, L. El, Bourais, I., Mohammadi, H., Amine, A., 2018. Recent advances in electrochemical biosensors based on enzyme inhibition for clinical and pharmaceutical applications. Sensors (Switzerland) 18. https://doi.org/10.3390/s18010164 
Holliger, P., Hudson, P.J., 2005. Engineered antibody fragments and the rise of single domains. Nat. Biotechnol. 23, 1126-1136. https://doi.org/10.1038/nbt1142

Hong-Geller, E., Micheva-Viteva, S., 2013. Small Molecule Screens to Identify Inhibitors of Infectious Disease, in: Drug Discovery. Los Alamos, pp. 157-175.

https://doi.org/http://dx.doi.org/10.5772/57353

Huang, X., Bai, Q., Pan, W., Hu, J., 2018. Quartz Crystal Microbalance with Approximately Uniform Sensitivity Distribution. Anal. Chem. https://doi.org/10.1021/acs.analchem.8b01529

Huang, X., Chen, Q., Pan, W., Hu, J., Yao, Y., 2019. Assessing the Mass Sensitivity for Different Electrode Materials Commonly Used in Quartz Crystal Microbalances (QCMs). Sensors (Basel). 19, 1-7. https://doi.org/http://dx.doi.org/10.3390/s19183968

Jiang, H., Jiang, D., Shao, J., Sun, X., 2016. Magnetic molecularly imprinted polymer nanoparticles based electrochemical sensor for the measurement of Gram-negative bacterial quorum signaling molecules ( $\mathrm{N}$-acyl-homoserine-lactones). Biosens. Bioelectron. 75, 411-419. https://doi.org/10.1016/j.bios.2015.07.045

Kim, M., Legido-Quigley, C., 2018. Small molecule biomarkers in Alzheimer's disease. Ocl 25, D404. https://doi.org/10.1051/ocl/2018027

Kim, Y.W., Sung, C., Lee, S., Kim, K.J., Yang, Y.H., Kim, B.G., Lee, Y.K., Ryu, H.W., Kim, Y.G., 2015. MALDI-MS-based quantitative analysis for ketone containing homoserine lactones in pseudomonas aeruginosa. Anal. Chem. 87, 858-863.

https://doi.org/10.1021/ac5039362

Kumar, J.S., Umesha, S., Prasad, K.S., Niranjana, P., 2016. Detection of Quorum Sensing Molecules and Biofilm Formation in Ralstonia solanacearum. Curr. Microbiol. 72, 297305. https://doi.org/10.1007/s00284-015-0953-0

Kumari, A., Pasini, P., Daunert, S., 2008. Detection of bacterial quorum sensing $\mathrm{N}$-acyl homoserine lactones in clinical samples. Anal. Bioanal. Chem. 391, 1619-1627. https://doi.org/10.1007/s00216-008-2002-3

Liu, J., Fu, K., Wang, Y., Wu, C., Li, F., Shi, L., Ge, Y., Zhou, L., 2017. Detection of Diverse $\mathrm{N}$-Acyl-Homoserine Lactones in Vibrio alginolyticus and Regulation of Biofilm Formation by N-(3-Oxodecanoyl) Homoserine Lactone In vitro. Front. Microbiol. 8, 1-15. https://doi.org/10.3389/fmicb.2017.01097

Mansouri, S., 2018. Miniaturization of Electrochemical Sensors, in: 17th International Meeting on Chemical Sensors - IMCS 2018. Bedford MA, pp. 252-253. https://doi.org/10.5162/IMCS2018/EC3.2

March, C., García, J. V., Sánchez, Á., Arnau, A., Jiménez, Y., García, P., Manclús, J.J., Montoya, Á., 2015. High-frequency phase shift measurement greatly enhances the sensitivity of QCM immunosensors. Biosens. Bioelectron. 65, 1-8. 
https://doi.org/10.1016/j.bios.2014.10.001

Martínez-Rivas, A., Chinestra, P., Favre, G., Pinaud, S., Séverac, C., Faye, J.C., Vieu, C., 2010. Detection of label-free cancer biomarkers using nickel nanoislands and quartz crystal microbalance. Int. J. Nanomedicine 5, 661-668.

https://doi.org/10.2147//JN.S12188

Middleton, B., Rodgers, H.C., CÃ $j$ mara, M., Knox, A.J., Williams, P., Hardman, A., Cámara, M., 2002. Direct detection of $\mathrm{N}$-acylhomoserine lactones in cystic fibrosis sputum. FEMS Microbiol. Lett. 207, 1-7. https://doi.org/10.1111/j.1574-6968.2002.tb11019.x Mitchell, J., 2010. Small molecule immunosensing using surface plasmon resonance. Sensors 10, 7323-7346. https://doi.org/10.3390/s100807323

Nimse, S.B., Sonawane, M.D., Song, K.S., Kim, T., 2016. Biomarker detection technologies and future directions. Analyst 141, 740-755. https://doi.org/10.1039/c5an01790d

Okano, C., Arai, M., Nasuno, E., limura, K., Morohoshi, T., Ikeda, T., Kato, N., 2012. Cyclodextrin Interaction with N -Hexanoyl Homoserine Lactone as Quorum Sensing Signal Produced in Gram-Negative Bacteria. Trans. Mat. Res. Soc. Japan 37, 315-318.

Ortori, C.A., Dubern, J.F., Chhabra, S.R., Cámara, M., Hardie, K., Williams, P., Barrett, D.A., 2011. Simultaneous quantitative profiling of $\mathrm{N}$-acyl-I-homoserine lactone and 2-alkyl$4(1 \mathrm{H})$-quinolone families of quorum-sensing signaling molecules using LC-MS/MS. Anal. Bioanal. Chem. 399, 839-850. https://doi.org/10.1007/s00216-010-4341-0 Piletska, E. V., Stavroulakis, G., Karim, K., Whitcombe, M.J., Chianella, I., Sharma, A., Eboigbodin, K.E., Robinson, G.K., Piletsky, S.A., 2010. Attenuation of vibrio fischeri quorum sensing using rationally designed polymers. Biomacromolecules 11, 975-980. https://doi.org/10.1021/bm901451j

Piletska, E. V., Stavroulakis, G., Larcombe, L.D., Whitcombe, M.J., Sharma, A., Primrose, S., Robinson, G.K., Piletsky, S.A., 2011. Passive control of quorum sensing: Prevention of pseudomonas aeruginosa biofilm formation by imprinted polymers.

Biomacromolecules 12, 1067-1071. https://doi.org/10.1021/bm101410q

Pimbley, D.W., Patel, P.D., 1998. A review of analytical methods for the detection of bacterial toxins. J. Appl. Microbiol. Symp. Suppl. 84, 98-109.

https://doi.org/10.1046/j.1365-2672.1998.0840s198s.x

Pirinçci, Ş.Ş., Ertekin, Ö., Laguna, D.E., Özen, F.Ş., Öztürk, Z.Z., Öztürk, S., 2018. Labelfree QCM immunosensor for the detection of ochratoxin A. Sensors (Switzerland) 18. https://doi.org/10.3390/s18041161

Poonguzhali, S., Madhaiyan, M., Sa, T., 2007. Production of acyl-homoserine lactone quorum-sensing signals is wide-spread in gram-negative Methylobacterium. J. Microbiol. Biotechnol. 17, 226-233.

Powers, R., Riekeberg, E., 2017. New frontiers in metabolomics: From measurement to 
insight. F1000Research 6. https://doi.org/10.12688/f1000research.11495.1

Prabowo, B.A., Purwidyantri, A., Liu, K.C., 2018. Surface Plasmon Resonance Optical Sensor: A Review on Light Source Technology. Biosensors 8.

https://doi.org/10.3390/bios8030080

Premaratne, G., Mubarak, Z.H.A., Senavirathna, L., Liu, L., Krishnan, S., 2017. Measuring ultra-low levels of nucleotide biomarkers using quartz crystal microbalance and SPR microarray imaging methods: A comparative analysis. Sensors Actuators, B Chem. 253, 368-375. https://doi.org/10.1016/j.snb.2017.06.138

Ronkainen, N.J., Halsall, H.B., Heineman, W.R., 2010. Electrochemical biosensors. Chem. Soc. Rev. 39, 1747-1763. https://doi.org/10.1201/b15589

Sauerbrey, G., 1959. Verwendung von Schwingquarzen zur Wägung dünner Schichten und zur Mikrowägung. Zeitschrift für Phys. 155, 206-222.

https://doi.org/10.1007/BF01337937

Selvolini, G., Marrazza, G., 2017. MIP-based sensors: Promising new tools for cancer biomarker determination. Sensors (Switzerland) 17. https://doi.org/10.3390/s17040718

Shankaran, D.R., Gobi, K.V., Miura, N., 2007. Recent advancements in surface plasmon resonance immunosensors for detection of small molecules of biomedical, food and environmental interest. Sensors Actuators, B Chem. 121, 158-177. https://doi.org/10.1016/j.snb.2006.09.014

Strausberg, R.L., Schreiber, S.L., 2003. From knowing to controlling: A path from genomics to drugs using small molecule probes. Science (80-. ). 300, 294-295. https://doi.org/10.1126/science.1083395

Susmel, S., Comuzzi, C., 2017. Selectivity and Efficiency of Conductive Molecularly Imprinted Polymer (c-MIP) Based on 5-Phenyl-Dipyrromethane and 5-PhenolDipyrromethane for Quorum Sensing Precursors Detection. Chemosensors 5, 5. https://doi.org/10.3390/chemosensors5010005

Taghadosi, R., Shakibaie, M.R., Masoumi, S., 2015. Biochemical detection of N-Acyl homoserine lactone ( $A H L)$ from biofilm-forming uropathogenic Escherichia coli (UPEC) isolated from urinary tract infection (UTI) samples. Reports Biochem. Mol. Biol. 3.

Uludağ, Y., Piletsky, S.A., Turner, A.P.F., Cooper, M.A., 2007. Piezoelectric sensors based on molecular imprinted polymers for detection of low molecular mass analytes. FEBS J. 274, 5471-5480. https://doi.org/10.1111/j.1742-4658.2007.06079.x

Van, Q.N., 2013. Current NMR Strategies for Biomarker Discovery, in: Proteomic and Metabolomic Approaches to Biomarker Discovery. pp. 87-117. https://doi.org/https://doi.org/10.1016/B978-0-12-394446-7.00006-6

Vashist, S.K., Vashist, P., 2011. Recent advances in quartz crystal microbalance-based sensors. J. Sensors 2011. https://doi.org/10.1155/2011/571405 
Wang, X., Chen, S., Jia, W., 2016. Metabolomics in Cancer Biomarker Research. Curr. Pharmacol. Reports 2, 293-298. https://doi.org/10.1007/s40495-016-0074-x

Wang, X.H., Wang, S., 2008. Sensors and biosensors for the determination of small molecule biological toxins. Sensors 8, 6045-6054. https://doi.org/10.3390/s8096045

Weiss, R., Freudenschuss, M., Krska, R., Mizaikoff, B., 2003. Improving methods of analysis for mycotoxins: Molecularly imprinted polymers for deoxynivalenol and zearalenone. Food Addit. Contam. 20, 386-395. https://doi.org/10.1080/0265203031000065827

Wöllner, K., Chen, X., Kremmer, E., Krämer, P.M., 2010. Comparative surface plasmon resonance and enzyme-linked immunosorbent assay characterisation of a monoclonal antibody with $\mathrm{N}$-acyl homoserine lactones. Anal. Chim. Acta 683, 113-118. https://doi.org/10.1016/j.aca.2010.10.015

Zhang, J., Yang, P.L., Gray, N.S., 2009. Targeting cancer with small molecule kinase inhibitors. Nat. Rev. Cancer 9, 28-39. https://doi.org/10.1038/nrc2559

Zheng, B., Cheng, S., Liu, W., Lam, M.H.W., Liang, H., 2013. Small organic molecules detection based on aptamer-modified gold nanoparticles-enhanced quartz crystal microbalance with dissipation biosensor. Anal. Biochem. 438, 144-149. https://doi.org/10.1016/j.ab.2013.03.030

Zhou W., Petricoin E.F., Longo C. (2017) Mass Spectrometry-Based Biomarker Discovery. In: Espina V. (eds) Molecular Profiling. Methods in Molecular Biology, vol 1606. Humana Press, New York, NY. https://doi.org/10.1007/978-1-4939-6990-6_19 